\title{
Connecting Mitochondria, Metabolism, and Stem Cell Fate
}

\author{
Anaïs Wanet, ${ }^{1}$ Thierry Arnould, ${ }^{1}$ Mustapha Najimi, ${ }^{2}$ and Patricia Renard ${ }^{1}$
}

As sites of cellular respiration and energy production, mitochondria play a central role in cell metabolism. Cell differentiation is associated with an increase in mitochondrial content and activity and with a metabolic shift toward increased oxidative phosphorylation activity. The opposite occurs during reprogramming of somatic cells into induced pluripotent stem cells. Studies have provided evidence of mitochondrial and metabolic changes during the differentiation of both embryonic and somatic (or adult) stem cells (SSCs), such as hematopoietic stem cells, mesenchymal stem cells, and tissue-specific progenitor cells. We thus propose to consider those mitochondrial and metabolic changes as hallmarks of differentiation processes. We review how mitochondrial biogenesis, dynamics, and function are directly involved in embryonic and SSC differentiation and how metabolic and sensing pathways connect mitochondria and metabolism with cell fate and pluripotency. Understanding the basis of the crosstalk between mitochondria and cell fate is of critical importance, given the promising application of stem cells in regenerative medicine. In addition to the development of novel strategies to improve the in vitro lineage-directed differentiation of stem cells, understanding the molecular basis of this interplay could lead to the identification of novel targets to improve the treatment of degenerative diseases.

\section{Introduction}

$\mathrm{S}$ TEM CELLS ARE DEFINED by two key properties: selfrenewal (ie, the ability to proliferate without lineage commitment) and pluripotency (ie, the ability to differentiate into more than one cell type) [1]. Careful regulation of these two properties is fundamental to ensure healthy development and tissue homeostasis. Stem cells can be sorted into three main categories: embryonic stem cells (ESCs), somatic (or adult) stem cells (SSCs), and induced pluripotent stem cells (iPSCs). ESCs arise from the inner cell mass of the early blastocyst and are the origin of all tissues derived from germ layers (endoderm, mesoderm, and ectoderm) during embryonic development. SSCs are found in different tissues of adult organisms, in which they ensure tissue homeostasis and response to injuries through the renewal of differentiated cells. The self-renewal and pluripotency abilities of SSCs are lower than those of ESCs. SSCs include, among others, hematopoietic stem cells (HSCs) and mesenchymal stem cells (MSCs). The third category of stem cells, iPSCs, are mature adult cells such as fibroblasts that have been artificially reprogrammed to an ESC-like state mainly through the overexpression of master stemness regulators, such as OCT4 (octamer-binding transcription factor 4), SOX2 (SRY (sex-determining region Y)box 2), KLF-4 (Kruppel-like factor 4), and $c-M y c$ [2].
Given the promising applications of stem cells in regenerative medicine and cell therapy, there is increasing interest in understanding the mechanisms regulating their self-renewal, pluripotency, and plasticity. Recent data support strong and direct involvement of mitochondria and oxidative metabolism in the regulation of stem cell pluripotency [3]. Cells adapt the number and activity of mitochondria in response to environmental and cellular cues through biogenesis, turnover, and fusion and fission processes [4]. Besides playing a fundamental role in energy production through oxidative phosphorylation (OXPHOS), mitochondria play important roles in amino acid, fatty acid, and steroid metabolism, as well as in cell signaling by reactive oxygen species (ROS) production, calcium homeostasis, and apoptosis [4].

The current review highlights the mitochondrial and metabolic changes that are associated with the differentiation of stem cells and the underlying pathways. We first describe the mitochondrial remodeling that occurs during pluripotent stem cell (PSC) differentiation and reprogramming, as well as the evidence, obtained by interfering with mitochondrial function, suggesting that mitochondria participate actively in those processes. We overview the recent data emerging from SSC studies suggesting that mitochondrial biogenesis and metabolic switches may be hallmarks of cell differentiation processes. Next, we address 3 questions: (i) What advantages does glycolysis bestow

\footnotetext{
${ }^{1}$ Laboratory of Biochemistry and Cell Biology (URBC), NAmur Research Institute for LIfe Sciences (NARILIS), University of Namur (UNamur), Namur, Belgium.

${ }^{2}$ Laboratory of Pediatric Hepatology and Cell Therapy, Institut de Recherche Clinique et Expérimentale (IREC), Université Catholique de Louvain, Brussels, Belgium.

(C) Anaïs Wanet et al., 2015; Published by Mary Ann Liebert, Inc. This Open Access article is distributed under the terms of the Creative Commons Attribution Noncommercial License (http://creativecommons.org/licenses/by-nc/4.0/) which permits any noncommercial use, distribution, and reproduction in any medium, provided the original author(s) and the source are credited.
} 
on PSCs? (ii) Are the mitochondrial and metabolic remodelings early or late events of stem cell differentiation/reprogramming? (iii) Can mitochondrial dynamics regulate the fate of stem cells? Finally, we provide an overview of the elucidated and putative molecular actors underlying the crosstalk between mitochondria, metabolism, pluripotency, and differentiation.

\section{Opposite Mitochondrial Remodeling and Metabolic Shifts During PSC Differentiation and Reprogramming}

Mitochondria display a characteristic ultrastructure. However, the first observations of mouse and human ESCs (mESCs and hESCs) using transmission electron microscopy surprisingly revealed immature, rare, and globular mitochondria displaying a perinuclear localization [5-10] and containing poorly developed cristae, as well as an electron-lucid matrix [11-15]. In contrast, somatic cells such as fibroblasts show mature elongated mitochondria, with numerous cristae and an electron-dense matrix [9] (Fig. 1). During the in vitro differentiation of hESCs, elongation of the mitochondrial network and maturation of the cristae ultrastructure are observed $[5,8,9,15]$. The reprogramming of human and mouse somatic cells into iPSCs results in opposite remodeling of the mitochondrial network in a process known as mitochondrial rejuvenation $[8,9,16]$. The ultrastructure, morphology, and intracellular distribution of mitochondria undergo reversible changes during the reprogramming of fibroblasts into iPSCs as well as subsequent redifferentiation into fibroblast-like cells, which support the importance of mitochondrial remodeling in differentiation and reprogramming events [8].

Besides morphological and ultrastructural changes, studies have observed an increase in mitochondrial DNA (mtDNA) content [15,17] and mass [8] during the differentiation of

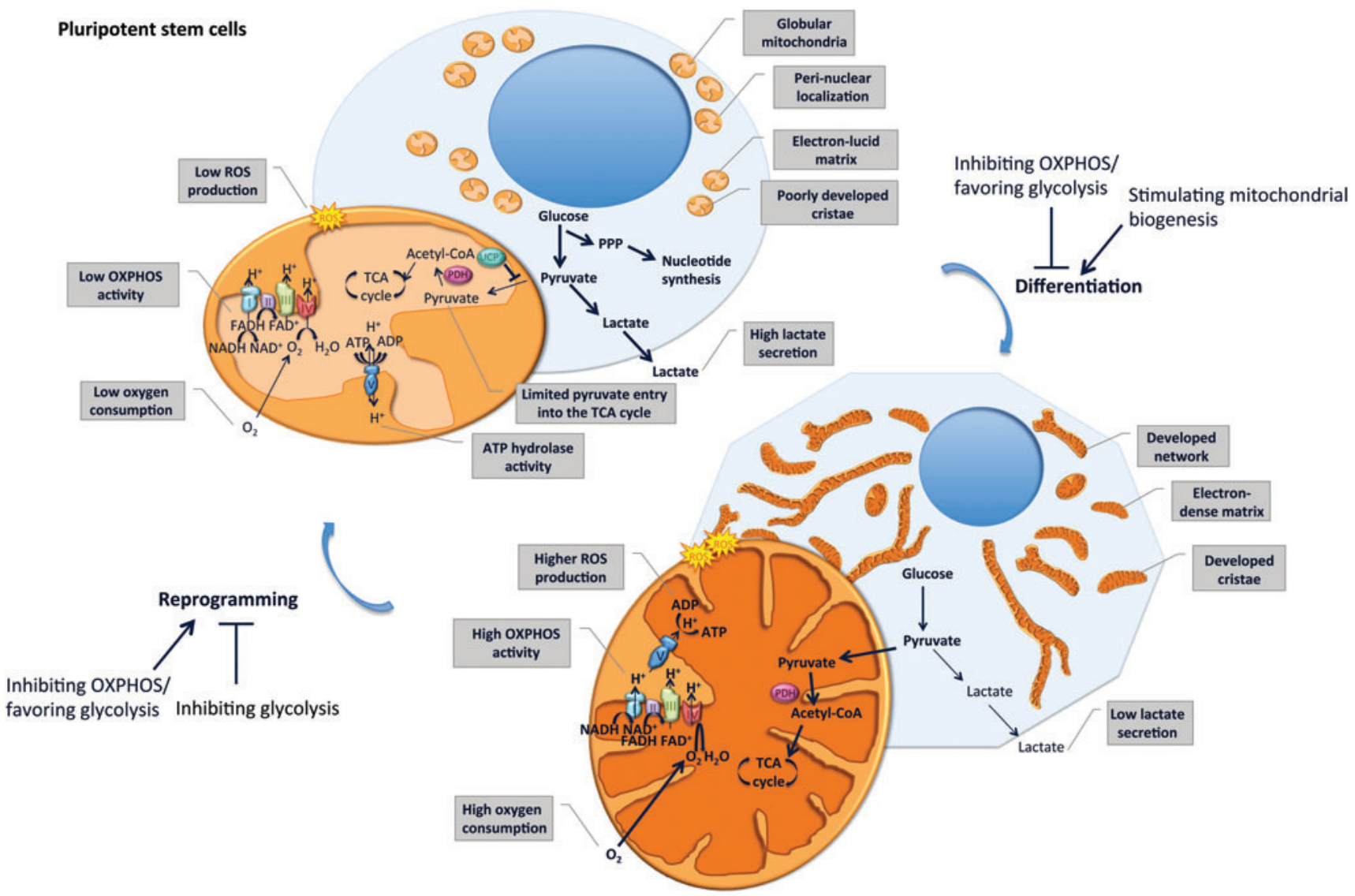

Differentiated cells

FIG. 1. Mitochondria and metabolism remodeling upon pluripotent stem cell differentiation and reprogramming. Pluripotent stem cells display globular immature mitochondria, localized perinuclearly, characterized by an electron-lucid matrix and poorly developed cristae. Energy production in pluripotent stem cells is mainly generated by a high glycolysis rate, leading to increased lactate production, whereas oxidative phosphorylation (OXPHOS) activity is limited, leading to reduced oxygen consumption and reactive oxygen species (ROS) production. Although substrate entry into the tricarboxylic acid (TCA) cycle is limited, intermediates of glycolysis enter the pentose phosphate pathway (PPP) and serve as substrates for the nucleotide synthesis required to sustain self-renewal. In differentiated cells, a more developed mitochondrial network, characterized by a more electron-dense matrix and developed cristae, ensures ATP production through increased OXPHOS activity. This results in elevated oxygen consumption and ROS production and reduced production of lactate through glycolysis. Interestingly, inhibiting OXPHOS or stimulating glycolysis impedes stem cell differentiation while favoring reprogramming into induced pluripotent stem cells (iPSCs). In contrast, stimulating mitochondrial biogenesis favors cell differentiation, whereas inhibiting glycolysis impairs reprogramming. Color images available online at www.liebertpub.com/scd 
hESCs, mESCs, and iPSCs. However, the evidence of a parallel increase in cell volume upon differentiation has moderated this apparent increase in mitochondrial content [18]. Further studies are needed to determine whether mitochondrial biogenesis reflects an adaptation to the increased cell volume upon differentiation.

In addition to the induction of mitochondrial biogenesis, the differentiation of PSCs, which collectively refer to ESCs and iPSCs, is associated with a metabolic shift, characterized by a transition from a predominant glycolysis-based metabolism in PSCs toward an increased OXPHOS-based metabolism in differentiated cells. Differentiated somatic cell lines display increased oxygen consumption rates, respiratory reserve capacity, and intracellular ATP content while secreting less lactate, indicative of reduced glycolysis, in comparison with hPSC lines [9]. Accordingly, the differentiation of hESCs, mESCs, and iPSCs is associated with increased intracellular ATP levels and lower lactate production, while reprogramming of somatic cells into iPSCs is accompanied by the reverse modifications [8,15,16,19]. Proteome remodeling, characterized by the induction of glycolytic enzymes and concomitant downregulation of multiple subunits of the mitochondrial complexes I and II, occurs during the reprogramming of mouse somatic cells into iPSCs [16].

Intracellular ATP levels of PSCs are more sensitive to inhibition of glycolysis than to inhibition of OXPHOS, whereas the opposite is observed in differentiated cells [18]. This observation supports glycolysis playing a predominant role in ATP production in hPSCs. Accordingly, the inhibition of OXPHOS with oligomycin, an $\mathrm{F}_{1} \mathrm{~F}_{0}$ ATP synthase inhibitor, decreases intracellular ATP by less than $5 \%$ in hPSCs, suggesting that minimal amounts of ATP are produced by OXPHOS in PSCs [18]. However, inducing mitochondrial uncoupling in hESCs, mESCs, and iPSCs using CCCP (carbonyl cyanide m-chlorophenylhydrazone) results in a drop in intracellular ATP levels and reduces proliferation rates. The AMP/ATP ratio increases in mESCs treated with the complex III inhibitor, antimycin A, suggesting that mESCs, at least partly, rely on OXPHOS for ATP production [20,21]. The discrepancies between the aforementioned studies might be explained by different degrees of OXPHOS inhibition. However, it has also been suggested that hPSCs may maintain the mitochondrial membrane potential through the ATP hydrolase activity of the dissociated $\mathrm{F}_{1} \mathrm{~F}_{0}$ ATP synthase components, thereby ensuring their proliferation and viability [18]. Thus, the decreased ATP content observed upon mitochondrial uncoupling in PSCs might be, at least partly, due to increased ATP hydrolase activity of the $\mathrm{F}_{1} \mathrm{~F}_{0}$ ATP synthase aimed at preserving the mitochondrial membrane potential.

Importantly, the mitochondrial ultrastructure and metabolism differ, depending on the pluripotency stage of PSCs. Two phases of pluripotency can be distinguished: naive and primed. Naive ESCs are derived from the preimplantation epiblast and display no differentiation bias unlike primed ESCs or epiblast stem cells (EpiSCs), which are derived from the postimplantation epiblast and represent a more mature stage [22]. Incidentally, recent data indicate that resetting hPSCs to a naive state (standard laboratory hESCs are similar to the mouse EpiSC stage) through transient overexpression of Nanog and KLF4 is associated with in- creased OXPHOS activity [23]. Furthermore, naive hESCs are less dependent on glycolysis for pluripotency maintenance than primed hESCs [23]. In mice, although EpiSCs were initially reported to be more glycolytic than mESCs, the latter displaying a bivalent metabolism [21], other studies documented that mouse EpiSCs are less glycolytic than mouse ESCs and iPSCs [24]. The use of cell lines versus primary cells may explain the discrepancies between those studies. At the morphological level, mouse EpiSCs contain more elongated mitochondria with more developed cristae than ESCs and iPSCs, although the mitochondria are still less mature than those of somatic cells [21,24]. These data emphasize that different levels of mitochondrial maturation and metabolism predilection exist, depending on the pluripotency stage. In the same vein, iPSCs maintained for extended in vitro culture periods display increased mitochondrial content and reduced potential to undergo neurogenesis in comparison with young iPSCs [25]. These data emphasize the importance of controlling the precise pluripotency stage and time in culture of stem cells in studies interested in their pluripotency and metabolic features.

\section{Modulating Metabolism or Mitochondrial Biogenesis Interferes with Stemness, Cell Differentiation, and Reprogramming}

Besides drawing simple correlations, several studies have found evidence for the direct involvement of mitochondria and metabolism in governing stemness and differentiation pathways. First, inhibiting mitochondrial function promotes pluripotency and prevents differentiation. Antimycin A, an inhibitor of mitochondrial respiratory complex III, promotes the expression of pluripotency markers, such as Nanog, while decreasing the mRNA abundance of genes related to differentiation in hESCs and mESCs [6,9,20]. Similarly, mitochondrial uncoupling by CCCP in hESCs and mESCs also increases the expression of Nanog, SOX2, and OCT4 and represses transcriptional programs necessary for embryonic lineage differentiation, such as $H O X$ genes [6]. In addition, the ectopic expression of the uncoupling protein 2 (UCP2) impairs the metabolic shift and hPSC differentiation [18].

Second, stimulating mitochondrial biogenesis reduces pluripotency and favors cell differentiation or commitment to specific lineages. The stimulation of mitochondrial biogenesis with S-nitrosoacetylpenicillamine (SNAP) in hESCs reduces the expression of pluripotency markers [10] and enhances the differentiation of mESCs into hepatocyte-like cells [26] and mESCs and hESCs into cardiomyocytes [10,27]. The overexpression of peroxisome proliferator-activated receptor (PPAR) gamma coactivator- $1 \alpha$ (PGC- $1 \alpha)$, the master regulator of mitochondrial biogenesis [28], in mouse iPSCs enhances their differentiation into adipocytes [29].

Third, favoring or inhibiting the metabolic shift toward glycolysis facilitates or prevents reprogramming into iPSCs, respectively, both in human and in mouse cells. The inhibition of glycolysis using 2-deoxy-D-glucose or oxalate in somatic cells prevents reprogramming into iPSCs, whereas compounds, such as glucose, D-fructose-6-phosphate, 2,4dinitrophenol (in response to OXPHOS uncoupling), and $\mathrm{N}$-oxaloylglycine, which stimulate glycolysis, enhance reprogramming [16,19,30]. Hypoxia, which stimulates glycolysis in response to the limited availability of oxygen, necessary 
for OXPHOS activity, preserves the self-renewal of hESCs and prevents their spontaneous differentiation $[31,32]$.

\section{Mitochondrial Biogenesis and Metabolic Switches as Apparent Hallmarks of Cell Differentiation Processes}

Mitochondrial biogenesis and the associated metabolic shift observed during the differentiation of PSCs appear to be hallmarks of differentiation processes, with various SSC differentiation models providing evidence for similar changes. An increased level of PGC- $1 \alpha$ mRNA, mitochondrial mass, and mtDNA copy number, together with increased formation of OXPHOS complexes and OXPHOS activity, is observed during the adipogenic differentiation of human bone marrow-derived MSCs (hBM-MSCs) [33-35]. Inhibiting mitochondrial biogenesis by the repression of PGC- $1 \alpha$ [36] or mitochondrial transcription factor A (TFAM) [33] impairs adipogenic differentiation, as does rotenoneinduced inhibition of mitochondrial respiration [33]. Similarly, the osteogenic differentiation of hBM-MSCs and umbilical cord-derived MSCs is accompanied by mitochondrial biogenesis, characterized by the induction of several mitochondrial biogenesis regulators, increased mtDNA copy number, cristae development, and elevated expression and activity of OXPHOS complexes [37-39]. We recently demonstrated increases in PGC- $1 \alpha$ expression, the mitochondria to cytoplasm ratio, mtDNA content, and OXPHOS expression and activity during the hepatogenic differentiation of hBMMSCs [40]. In mouse HSCs (mHSCs), the mitochondrial mass increases during the early stages of differentiation [41], whereas mitochondrial respiration defects, caused by mitochondrial DNA depletion, impair their differentiation [42].

Similarities might exist in the mitochondrial content and function of normal and cancer stem cells (CSCs) as lung CSCs display reduced mitochondrial content and function compared with non-CSCs [43]. Nasopharyngeal carcinoma CSCs contain round-shaped perinuclear mitochondria similar to those observed in ESCs as well as increased glycolytic metabolism compared with their differentiated counterparts. In addition, blocking the glycolytic pathway alters the properties of CSCs [44]. These observations support the idea that relationships between mitochondria, metabolism, and stemness may underlie both physiological and pathological conditions.

\section{Glycolysis Benefits PSCs}

Glycolytic metabolism is less efficient than OXPHOS in terms of the energy production yield, raising questions as to why PSCs might favor this type of metabolism. First, a high glycolysis rate, combined with reduced OXPHOS, might be necessary for PSCs to supply the cofactors and substrates necessary for the biosynthetic reactions underlying their expansion [45]. In line with this hypothesis, the proliferative capacity of mESCs is associated with high activity of different glycolytic enzymes, elevated glycolytic flux, and low mitochondrial oxygen consumption [46]. When compared with differentiated cells, hPSCs display more phosphorylated pyruvate dehydrogenase (PDH) E1 $\alpha$ [9], which inactivates the PDH complex and results in lower levels of substrates entering the tricarboxylic acid (TCA) cycle [47].
Similarly, the expression of pyruvate dehydrogenase kinase (PDK) 2 and 4, which prevents the mitochondrial oxidation of pyruvate through the inhibition of the PDH complex and favors glycolysis, is important for the quiescence and function of mHSCs [48]. UCP2 prevents mitochondrial glucose oxidation through a substrate-shunting mechanism, resulting in increased glycolysis and nucleotide synthesis through the pentose phosphate pathway [49]. UCP2 is highly expressed in hPSCs compared with differentiated cells. Its ectopic expression, which is normally repressed upon differentiation of PSCs, prevents any metabolic shift and impairs their differentiation [18]. PSCs also express higher mRNA levels of transketolase. Transketolase connects glycolysis to the biosynthetic pentose phosphate pathway, which is involved in nucleotide synthesis [50]. Therefore, PSCs increase the flux of glycolysis intermediates entering the pentose phosphate pathway while inhibiting their entry into the TCA cycle, thus favoring nucleotide synthesis over energy production.

The second advantage for PSCs in circumventing oxidative metabolism might be to reduce the production of ROS, which can damage genetic material and cell constituents. Maintaining low levels of ROS could be of critical importance for organismal development and homeostasis in providing protection against such damage. In contrast, increasing levels of ROS might be necessary for the commitment to specific cell types by activating particular signaling pathways, as discussed later (see The tight regulation of cell fate by ROS section).

Third, as PSCs reside in hypoxic niches, their glycolytic metabolism might represent an adaptation to their environment. In agreement with this hypothesis, hMSCs, when cultured under normoxia $\left(21 \% \mathrm{O}_{2}\right)$, can use OXPHOS efficiently. However, expansion under $21 \% \mathrm{O}_{2}$ increases senescence in MSCs when compared with physiological oxygen levels $\left(5 \% \mathrm{O}_{2}\right)$, suggesting that a glycolysis-based metabolism favored by a hypoxic environment might protect MSCs against senescence [51].

\section{Mitochondria and Metabolism Remodeling as Early Events in Cell Differentiation and Reprogramming Processes}

Mitochondrial biogenesis and metabolic shifts are early events in multiple stem cell differentiation models, with most changes observed in the first stage of the differentiation process. Maturation of the mitochondrial network, as well as increased transcription of mtDNA, is observed during the differentiation of hESCs into cardiomyocytes [5]. The development of the mitochondrial network precedes the loss of the pluripotency markers, OCT4 and Nanog, in differentiating hESCs [6]. Mitochondrial biogenesis and metabolic shift toward OXPHOS are also early events in osteogenic [37,39], adipogenic [34], and hepatogenic [40] differentiation of hMSCs. In mHSCs, the upregulation of mitochondrial biogenesis was demonstrated to parallel the loss of pluripotency [41]. Mitochondrial respiration is more important in the commitment of mHSCs into lineagerestricted progenitor cells than in reaching the final phase of cell differentiation [42].

In contrast, the metabolic reprogramming observed during the generation of iPSCs occurs before the reestablishment of a pluripotency status [16]. Favoring glycolysis 
during the first days of reprogramming of human somatic cells is sufficient to increase the efficiency of the reprogramming, although no iPSCs are derived at the end of glycolysis-stimulating treatment [30]. These data suggest that enhanced glycolysis might predispose somatic cells to more efficient reprogramming into iPSCs. Consistently, the bioenergetic status of different somatic cells correlates with their reprogramming efficiencies: the closer the metabolic status of human somatic cells resembles that of PSCs, the better and faster is the reprogramming efficiency [19]. Although a glycolytic-based metabolism represents a prerequisite for efficient iPSC generation, continuous remodeling of the metabolic status of iPSCs occurs during the reprogramming time course. Mouse iPSCs more closely resemble ESCs when cultured for longer periods [52]. Similarly, in humans, the metabolome of late-passaged iPSCs (passage 41 and over) is more comparable with ESCs than that of early passaged iPSCs (passage 16) [19].

\section{A New Shape for a New Fate: Mitochondrial Dynamics as a Regulator of Cell Fate}

Mitochondrial dynamics influence most, if not all, mitochondrial-dependent biological processes, such as apoptosis, calcium homeostasis, and ATP production. Emerging evidence also points toward a role for mitochondrial fusion and fission in signaling pathways regulating stem cell proliferation and differentiation [3]. Mitochondrial elongation requires the fusion of the outer and inner mitochondrial membranes (OMM and IMM). The dynamin-related GTPases, mitofusin 1 (MFN1) and 2 (MFN2), mediate the fusion of the OMM, and the optic atrophy 1 (OPA1) protein and MFN1 mediate the fusion of the IMM. Several other proteins are involved in mitochondrial fusion, including prohibitin (PHB), which regulates OPA1 processing [53]. The cytosolic dynamin-related protein 1 (DRP1) regulates mitochondrial fission. Upon recruitment to the OMM by receptors, including the mitochondrial fission factor (MFF), the mitochondrial dynamic proteins of $49 \mathrm{kDa}$ (MID49) and $51 \mathrm{kDa}$ (MID51), and mitochondrial fission 1 (FIS1), DRP1 oligomerizes and induces mitochondrial constriction and fission [54]. Multiple post-translational modifications are involved in the regulation of the mitochondrial dynamic machinery $[54,55]$.

Reduced expression 1 ( $R E X 1)$, a widely used pluripotency marker, was only recently found to regulate cell fate through its effect on mitochondrial dynamics [56]. Repressing REX1 expression in hPSCs results in the downregulation of pluripotency markers, upregulation of differentiation markers of all three germ layers, and development of mitochondrial morphology and activity. Mechanistically, these effects are mediated by the positive regulation of cyclin $\mathrm{B} 1 / \mathrm{B} 2$ by REX1. This leads to increased cyclin B/cyclin-dependent kinase 1-dependent phosphorylation of DRP1 at Ser616, a post-translational modification triggering mitochondrial fission. Accordingly, the overexpression of REX1 or the DRP1S616D mutant protein favors reprogramming into iPSCs, whereas repressing the expression of REX1 or DRP1 prevents reprogramming [56]. Although these data suggest a critical role for DRP1-mediated mitochondrial fission in the pluripotency of hESCs, studies of mESCs reported contradictory results. In mouse embryonic fibroblasts, although pharmacological inhibition of the self-assembly of DRP1 reduces the reprogramming efficiency [57], the knockdown of DRP1 expression does not affect pluripotency gene expression [58]. In addition, the increased levels of DRP1 resulting from the depletion of growth factor erv1-like, a flavin adenine dinucleotide-dependent sulfhydryl oxidase predominantly found in the intermembrane space of mitochondria, inhibit the expression of pluripotency markers in mESCs [59]. The discrepancies in the relationship of DRP1 with pluripotency might be due to species-specific responses. For example, overexpressing REX1 does not confer any advantageous effect during the reprogramming of mouse fibroblasts [56]. Although mainly considered as a component of the mitochondrial fission machinery, DRP1 can also promote mitochondrial fusion by its interaction with MFN2 [60]. The knockdown of DRP1 leads to the elongation of the mitochondrial network of fibroblasts, but it does not cause substantial changes in the morphology of mouse ESC/iPSC mitochondria [58]. These findings suggest that the discrepancies between the previous studies might also depend on DRP1 playing a different role in mitochondrial dynamics in different cells. Furthermore, tight control of DRP1 levels and/or activity, possibly regulated by different post-translational modifications, might underlie these observations. Beyond its involvement in pluripotency, DRP1-mediated fission is required for the terminal differentiation of mESCs in neuronal lineages [58] as well as for the myogenic differentiation of myoblasts [61].

Prohibitin 2 (PHB2) was recently shown to be involved in the control of stem cell fate. Overexpression of PHB2, which is normally high in mESCs, favors the proliferation of mESCs, but inhibits their differentiation into neuronal and endodermal lineages. The overexpression of PHB2 also prevents the maturation of mitochondria observed during mESC differentiation [62]. The effect of PHB2 on the maturation of mitochondria is mediated by enhanced OPA1 processing, resulting in increased mitochondrial fission and dysfunctional mitochondria [62]. The depletion of protein tyrosine phosphatase, mitochondrial 1 (PTPMT1), a mitochondrial phosphatidylinositol phosphate (PIP) phosphatase, reduces oxidative metabolism, increases glycolysis in mESCs, and prevents their differentiation. These effects are mediated by increased mitochondrial fission due to the accumulation of PIP substrates in the mitochondria [63]. Interestingly, PTPMT1 is also required for the differentiation of mHSCs. PTPMT1 acts by priming mitochondria for a rapid metabolic transition toward OXPHOS. Mechanistically, the accumulation of PIP substrates resulting from the depletion of PTPMT1 induces the activity of UCP2, which, as mentioned earlier, inhibits pyruvate oxidation in mitochondria and thus impairs metabolic shifts [64]. An increase in MFN2 and OPA1 expression is required for the differentiation of mESCs into the cardiomyocyte lineage, supporting the involvement of mitochondrial fusion in the differentiation of mESCs. Inhibiting mitochondrial fusion by repressing the expression of MFN2 and OPA1 in mESCs results in the activation of the $\mathrm{Ca}^{2+}$ /calcineurin signaling pathway, leading to increased Notch activity and the repression of myocyte enhancer factor2c and GATA-binding protein 4, two cardiomyocyte lineage transcriptional regulators [65].

Together, the aforementioned mechanistic studies demonstrate that mitochondrial dynamics, encompassing both fusion and fission events, are involved both in cell differentiation 
and in reprogramming events. The bidirectional influences of mitochondrial dynamics on pluripotency and differentiation are predictable because pluripotency appears to affect mitochondrial dynamics and activity, which regulates pluripotency and differentiation, as outlined in the following sections. As recently reviewed elsewhere [66], crosstalk also exists between cell cycle regulators and the components of mitochondrial dynamics. As cell cycle exit is generally required in differentiation programs, this crosstalk might also be involved in the regulation of cell differentiation.

\section{Molecular Crosstalk Between Mitochondria, Metabolism, Pluripotency, and Differentiation}

Various models have firmly established the differences in the mitochondria and metabolism of pluripotent and differentiated cells. Efforts are now underway to unveil how pluripotency and differentiation affect mitochondrial biogenesis and function and how mitochondria and metabolism modulate pluripotency and differentiation. In the following sections, we describe the pathways involved in the bidirectional crosstalk between mitochondria and metabolism and the regulation of pluripotency and differentiation (Fig. 2).

\section{Oxygen sensing and the HIF pathway}

Oxygen sensing plays a central role in metabolic homeostasis and is partly regulated by the hypoxia-inducible factor (HIF) family of transcription factors. HIF-1 is a heterodimer containing a constitutively expressed $\beta$ subunit as well as an oxygen-regulated $\alpha$ subunit that is continuously synthesized and then degraded by prolyl hydroxylases under normoxia. In response to hypoxia, HIF-1 activation promotes a switch from mitochondrial respiration to anaerobic glycolysis and suppresses mitochondrial biogenesis [67]. Interestingly, most stem cell types reside in hypoxic niches, and increasing evidence points toward a critical role for oxygen in the regulation of the function of stem cells [68]. Thus, it is not surprising that several lines of evidence support a role for HIF-1 in the interplay between mitochondria, metabolism, and stem cell functions. As HIF-1 is involved in the dedifferentiation of cancer cells [69], it might also regulate differentiation pathways in pathological conditions.

The protein kinase $\mathrm{C}$ isoform lambda/iota $(\mathrm{PKC} \lambda / \mathrm{l})$ is involved in the coregulation of mitochondrial biogenesis and stem cell pluripotency/differentiation through the HIF-1 $\alpha$ pathway [70]. The depletion of $\mathrm{PKC} \lambda / \mathrm{l}$ induces a

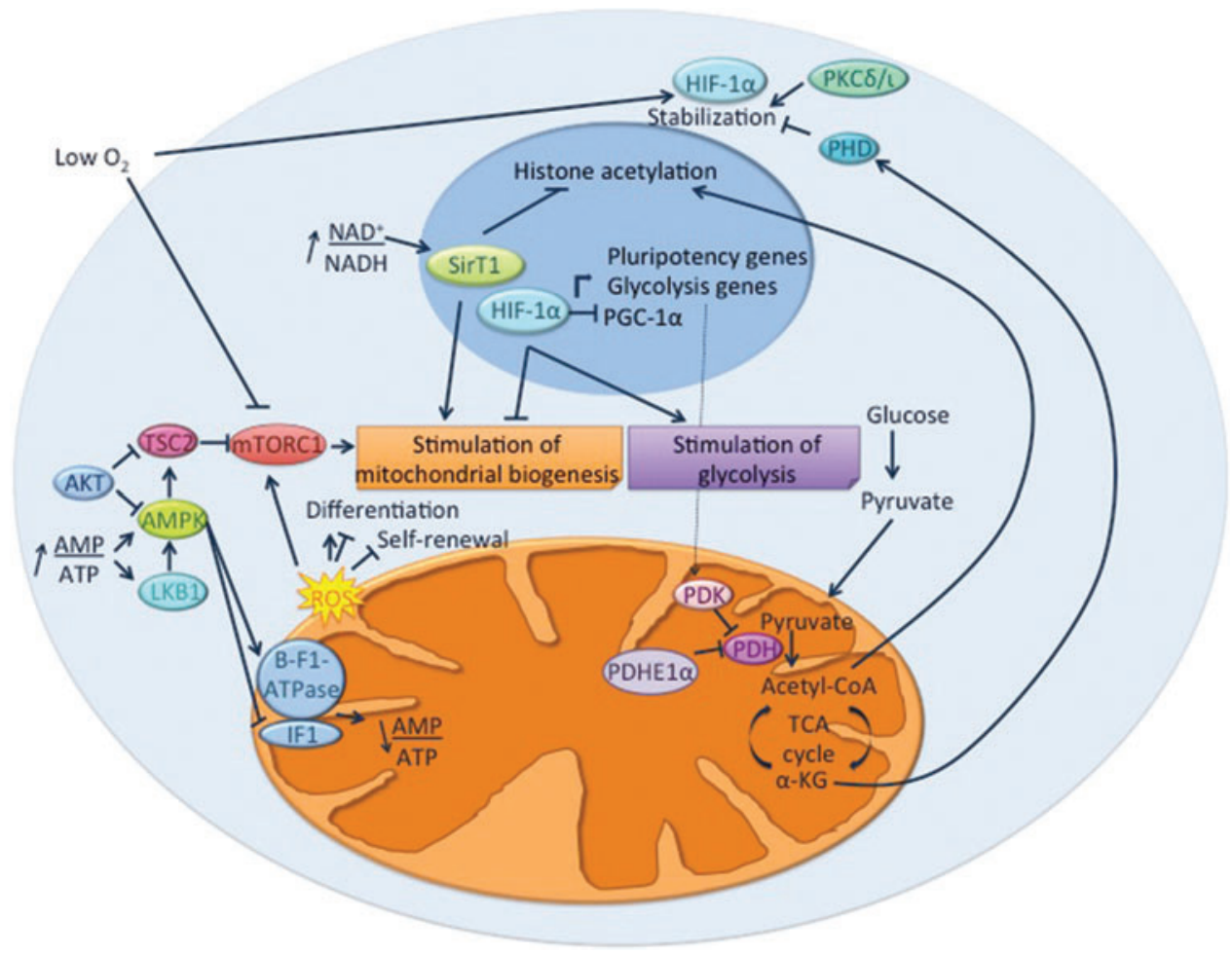

FIG. 2. Pathways involved in the interplay between pluripotency, mitochondrial biogenesis, and metabolism. On the one hand, energy-, nutrient-, and environment-sensing pathways regulate both pluripotency and metabolism through their effects on glycolysis and mitochondrial biogenesis and activity. On the other hand, mitochondrial activity regulates stemness and differentiation through various mechanisms. These involve, for example, the production of ROS, which can induce or prevent the commitment to specific differentiation lineages; the production of intermediates or cofactors influencing epigenetic marks, protein activity, and stability; and the modification of the redox or energy status of the cells, thus altering nutrient- and energy-sensing pathways. AMPK, AMP-activated protein kinase; IF1, inhibitory factor I; LKB1, liver kinase B1; HIF-1 $\alpha$, hypoxia-inducible factor $1 \alpha$; mTORC1, mammalian target of rapamycin complex I; PDH, pyruvate dehydrogenase; PDK, pyruvate dehydrogenase kinase; PGC- $1 \alpha$, peroxisome proliferator-activated receptor gamma coactivator $1 \alpha$; PHD, prolyl hydroxylase domain-containing protein; PKC $\lambda / 1$, protein kinase $\mathrm{C}$ isoform lambda/iota; TCA, tricarboxilic acid; TSC2, tuberous sclerosis complex 2; $\alpha-K G, \alpha$-ketoglutarate. Color images available online at www.liebertpub.com/scd 
metabolic shift, resulting in increased glycolysis and decreased OXPHOS activity. It also promotes naive pluripotency in mESCs. These effects are mediated by stabilization of HIF- $1 \alpha$, which positively regulates the expression of pluripotency genes while suppressing the expression of PGC- $1 \alpha$ by direct binding to their promoters. Thus, HIF- $1 \alpha$ simultaneously regulates pluripotency and mitochondrial biogenesis by activating and repressing transcriptional activities, respectively [70].

The involvement of HIF- $1 \alpha$ in pluripotency is not limited to ESCs. Upon reprogramming of human cells, HIF-1 $\alpha$ improves the induction of pluripotency by activating a glycolytic program, predisposing the somatic cells to more efficient reprogramming [71]. The expression of HIF-1 $\alpha$ is also important for the quiescence and function of mHSCs. It maintains the expression of PDK, which, by inactivating the PDH complex, prevents the mitochondrial oxidation of acetyl-CoA and favors glycolysis. On the other hand, HIF$1 \alpha$ deficiency results in a shift from glycolysis to OXPHOS and is associated with increased mitochondrial mass in HSCs [48]. In hMSCs, hypoxia or the stabilization of HIF$1 \alpha$ using cobalt chloride prevents the osteogenic differentiation and the associated increase in mitochondrial biogenesis [72].

Thus, HIF- $1 \alpha$ can control pluripotency gene expression and promote glycolytic metabolism while inhibiting mitochondrial biogenesis and function. It remains to be determined whether PKC $\lambda / 1$ acts systematically upstream of HIF$1 \alpha$. Other questions that need to be addressed are whether other upstream regulators are involved in the activation of HIF- $1 \alpha$ and whether such activation depends on cell types or lineage commitment. In addition, the roles of other molecular actors (ie, whether they act together or independently of HIF-1 $\alpha$ ) in the control of pluripotency gene expression and activation of glycolysis need to be determined.

\section{The tight regulation of cell fate by ROS}

ROS are highly reactive reduced forms of molecular oxygen, such as the superoxide radical anion $\left(\mathrm{O}_{2}{ }^{-}\right)$and hydrogen peroxide $\mathrm{H}_{2} \mathrm{O}_{2}$. Under physiological conditions, ROS are naturally produced by the respiratory chain during OXPHOS [73]. Although ROS exert oxidative damage on lipids, proteins, and DNA, they also act as secondary messengers and were recently shown to be involved in the regulation of stem cell self-renewal, pluripotency, and differentiation [74].

Although a specific role for mitochondrial ROS has yet to be demonstrated in ESCs, stimulating or inhibiting the production of ROS favors or prevents mESC differentiation into cardiomyocytes, respectively [75-79]. However, it needs to be emphasized that the stimulating effect of ROS on cardiomyocyte differentiation might strongly depend on the level and nature of the ROS. Indeed, mESCs simultaneously treated with antioxidants and cyclosporin $\mathrm{A}$, an inhibitor of the mitochondrial permeability transition pore (mPTP) resulting in increased mitochondrial biogenesis, activity, and ROS generation, synergistically favor cardiomyocyte differentiation [80].

ROS regulate the proliferation and differentiation of rat neural progenitors, with proliferating neural progenitors displaying low levels of ROS in comparison with their differentiated neuronal and astrocytic counterparts [81]. A recent study showed that rapid bursts of superoxide radical anions called superoxide flashes, which are highly reactive reduced forms of molecular oxygen, were involved in regulating the self-renewal and differentiation of mouse embryonic neural progenitor cells (NPCs). Mitochondrial superoxide scavengers or MPTP inhibitors reduce the frequency of superoxide flashes and enhance NPC proliferation [82]. In contrast, increased superoxide flashes are necessary for the differentiation of mouse NPCs into cortical neurons, and inhibiting mPTP or scavenging mitochondrial ROS impairs differentiation [83]. In a mouse model of adult hippocampal neurogenesis, a peak in mitochondrial abundance and ROS levels was observed in a highly proliferative, intermediate progenitor state, but not in undifferentiated neural stem cells or postmitotic neurons [84]. Although these differences might depend on the embryonic or adult status of the NPCs, the data suggest that the involvement of ROS in neurogenesis might be limited only to a specific stage of cell differentiation.

In hMSCs, mitochondrial complex III-derived ROS, independently of OXPHOS, are required for the expression of PPAR $\gamma$ and initiation of the adipogenic transcriptional program [34]. Mitochondria-targeted antioxidants such as mitoCP prevent both the adipogenic differentiation and the associated increase in ROS, whereas the addition of exogenous $\mathrm{H}_{2} \mathrm{O}_{2}$ to mitoCP-treated hMSCs rescues adipogenic differentiation [34]. Similarly, hypoxia stimulates adipocyte differentiation by enhancing the production of mitochondrial ROS [85]. On the other hand, both exogenous $\mathrm{H}_{2} \mathrm{O}_{2}$ and oligomycin-dependent inhibition of mitochondrial activity inhibit the osteogenic differentiation of hMSCs. These data suggest that mitochondrial OXPHOS is required for osteogenic differentiation and that excessive ROS hamper osteogenic differentiation [37]. Beyond their role in MSC differentiation, mitochondrial ROS are also involved in MSC senescence. The treatment of mouse MSCs with transforming growth factor $\beta 1$ induces their senescence. This effect is, at least partly, mediated by the generation of mitochondrial ROS, suggesting that maintaining low levels of mitochondrial ROS is necessary to preserve MSC stemness [86].

The involvement of ROS in the function of HSCs has also been established and recently reviewed [74,87]. mHSCs with low ROS levels are more quiescent and display increased self-renewal potential compared with HSCs displaying higher ROS levels, which are prone to exhaustion upon serial transplantations [88]. The negative impact of ROS on mHSC self-renewal is mediated by the activation of p38 MAPK and mammalian target of rapamycin (mTOR) pathways, with studies showing that inhibiting either of these pathways restores the long-term reconstitution ability of HSCs with high levels of ROS [88,89]. In contrast, ROS triggered the differentiation of a Drosophila population of hematopoietic progenitors resembling mammalian myeloid progenitors through the activation of c-Jun N-terminal kinases, leading to activation of Forkhead box $\mathrm{O}$ and inhibition of the activity of polycomb [90]. Similarly, increasing ROS in AKT1/AKT2 double-deficient mHSCs, which show lower ROS levels, rescues the defects in differentiation [91]. Thus, low levels of ROS are necessary to preserve the quiescence of HSCs, and increased ROS levels favor HSC differentiation. 
The generation of mitochondrial ROS may also represent a key upstream signaling event in epidermal differentiation and hair follicle development [92]. In mouse keratinocytes, preventing the generation of mitochondrial ROS through the ablation of TFAM, which is required for the transcription of mtDNA-encoded mitochondrial genes [93], disturbs the transduction of Notch signaling required for keratinocyte differentiation [92].

Based on the aforementioned, maintaining low ROS levels, notably through reduced mitochondrial activity, appears necessary to preserve the self-renewal ability of different types of stem cells, and increasing ROS seems to favor particular lineage commitment during differentiation. Maintaining low ROS levels in stem cells, possibly through reduced mitochondrial function, might represent a protective mechanism of the genome against ROS-mediated damage, which is essential to ensure optimal development and organism homeostasis upon aging. Further studies are required to elucidate more precisely which type(s) of ROS are endogenously produced in different models of stem cell differentiation and whether different types of ROS exert similar or opposite effects on pluripotency and differentiation in various models.

\section{Nutrient- and energy-sensing pathways: a complex connection between energy metabolism and stem cell function}

Given that stem cells and differentiated cells display different metabolisms and metabolomes, it is not surprising that nutrient- and energy-sensing pathways are involved in the regulation of stem cell pluripotency and differentiation. The following sections illustrate the roles of major sensing pathways in orchestrating the interplay between mitochondria, metabolism, stem cell pluripotency, and differentiation.

The mTOR pathway. The mTOR pathway plays a crucial regulatory role in cell metabolism, proliferation, growth, and survival by sensing extracellular and intracellular signals, such as oxygen, and the energy status, growth factors, and amino acid content. Two distinct complexes, mTORC1 and mTORC2, exist and exert overlapping but distinct functions. The mTORC1 complex is sensitive to amino acids, stress, oxygen, energy, and growth factors. It promotes cell growth and cell cycle progression by inducing anabolic processes, such as the biosynthesis of proteins and lipids and the stimulation of mitochondrial biogenesis and metabolism, while limiting catabolic processes, including autophagy. The mTORC2 complex, which has not been described in as much detail as the mTORC1 complex, responds to growth factors and regulates metabolism, cell survival, and cytoskeletal organization [94,95]. The mTORC1 complex is acutely sensitive to rapamycin treatment [95]. In contrast, the mTORC2 complex is insensitive to acute rapamycin treatment, although chronic exposure disrupts the mTORC2 pathway [95]. The mTORC1 pathway is involved in the regulation of pluripotency and differentiation, notably through its relationship with mitochondrial biogenesis.

The involvement of mTORC1 in ESC pluripotency and differentiation appears complex. On the one hand, mTOR activity is required for the proliferation of hESCs and mESCs [96] and its inhibition impairs pluripotency in
hESCs [97]. The sorting of mESCs according to low or high mitochondrial membrane potential also reflects the activation status of mTORC1 in ESCs and is correlated with pluripotency and differentiation capacities [98]. Treatment with rapamycin decreases pluripotency and increases the differentiation capacities of mESCs with high mitochondrial membrane potential [98]. On the other hand, Deptor, an endogenous inhibitor of mTORC1 and mTORC2 activity, maintains mESC pluripotency, and repression of Deptor triggers differentiation [99]. Increased mTORC1 activity has been consistently shown during the differentiation of mESCs and hESCs into a neuronal lineage $[99,100]$ and the differentiation of hESCs into fibroblast-like cells [101]. In mice, rapamycin-induced inhibition of mTOR activity increases the reprogramming efficiency [102], whereas the overactivation of the mTOR pathway in cells lacking tuberous sclerosis complex 2, a negative regulator of mTORC1 [95], impairs reprogramming [103]. Likely, tight control of mTOR signaling determines the outcome of pluripotency and differentiation, possibly with differences related to lineages, species, and the relative contribution of mTORC1 and mTORC2 signaling. In agreement with this hypothesis, treatment of hESCs with $100 \mathrm{nM}$ rapamycin for 6 days results in a loss of the pluripotency markers, OCT-4, Nanog, and SOX2 [97], whereas treatment with $20 \mathrm{nM}$ rapamycin for 3 days has no impact on pluripotency gene expression [101]. The differences in mTOR activity may also be due to the precise stage of pluripotency (naive vs. primed ESCs, or ESCs vs. epiSCs), with naive ESCs having increased mTOR activity and mitochondrial biogenesis and function compared with primed ESCs/epiSCs.

The involvement of mTOR has also been studied in SSCs. Reduced activity of mTORC1 signaling preserves mHSC self-renewal and differentiation potential by repressing mitochondrial biogenesis and ROS production [104]. Nonetheless, mTORC1 activity is still required for mHSC function as the deletion of the regulatory-associated protein of mTOR (Raptor) ablates mTORC1 activity and results in defective hematopoiesis [105]. There also seems to be a link between mTORC1 signaling and stem cell function, partly dependent on ROS, in hMSCs. During the adipocyte differentiation of hMSCs, mTORC1 is required for the generation of ROS, which is also required for the differentiation process (see The tight regulation of cell fate by ROS section). Although overexpression of PPAR $\gamma$ facilitates adipogenesis in the presence of antioxidants, this effect is lost upon PI3K or mTORC1 inhibition, suggesting that mTORC1 additionally favors adipocyte differentiation by effectors other than ROS [34].

Collectively, these data suggest that the magnitude and duration of mTORC1 activation or inhibition, rather than an on/off status, likely influence stem cell pluripotency and differentiation. Different effectors downstream of mTORC1 signaling might be involved, depending on the stem cell type and differentiation lineages. Most studies have focused on mTORC1. The contribution of mTORC2 remains elusive and should be the focus of further experiments. Given the central role of metabolism in regulating stemness and differentiation, it is important to investigate the effectors that connect the activity status of mTOR with stemness and differentiation.

$A M P K$ and its upstream regulators. AMP-activated protein kinase (AMPK) is a key heterotrimeric, intracellular energy 
sensor sensitive to the AMP/ATP ratio. In low-energy conditions (a high AMP/ATP ratio), AMPK is activated and induces catabolic pathways, such as fatty acid oxidation or glucose uptake, while inhibiting anabolic pathways, including lipid and protein synthesis, to restore the energy balance [106].

Downregulated expression of the catalytic $\alpha 1$ subunit of AMPK is found in hESCs and iPSCs when compared with differentiated cells [107]. Interestingly, metformin-activated AMPK impedes human and mouse iPSC generation through the establishment of a metabolic barrier distinct from the enhanced glycolysis required for reprogramming [108]. Mechanistically, the activation of AMPK upregulates the catalytic $\beta$-F1-ATPase subunit, a rate-limiting component of mitochondrial OXPHOS normally repressed upon reprogramming. Simultaneously, AMPK activation prevents the induction of the ATPase inhibitory factor 1, thus impeding the metabolic shift required for reprogramming [109]. However, the role of AMPK in pluripotency appears very complex as the activation of AMPK in mESCs is associated with upregulation of pluripotency genes [110]. Thus, AMPK activity is likely not directly involved in the promotion of pluripotent versus differentiated cells. Instead, the intracellular environment, comprising a precise metabolome and proteome at a given moment, as well as the activation of different effectors, likely influences the outcome of AMPK activity in determining the fate of stem cells. Accordingly, the involvement of AMPK in cell differentiation differs, depending on the lineage commitment. AMPK activity inhibits mouse myoblast differentiation [111], whereas it favors human endothelial [112] and mouse cardiac [113] differentiation. In the latter model, the simultaneous knockdown of several adenylate kinase isoforms normally induced during cardiogenesis decreases AMPK activity and impedes cardiomyocyte differentiation and associated mitochondrial biogenesis [113]. The production of nitric oxide, a key regulator of cardiomyogenesis in mESCs and potent inducer of mitochondrial biogenesis, occurs downstream of AMPK and mTORC1 signaling pathways [114]. Thus, these two pathways may interact to control the interplay between mitochondria, metabolism, stemness, and differentiation.

AMPK is also involved in the regulation of SSC pluripotency and differentiation. As recently reviewed elsewhere, AMPK activity controls the fate of MSCs by favoring osteogenic and inhibiting adipogenic differentiation. The role of AMPK in controlling the differentiation of MSCs may depend on its involvement in multiple pathways, including the ERK pathway, mTOR signaling, Wnt/ $\beta$ catenin signaling, and energy metabolism [115]. In mHSCs, the constitutively active liver kinase B1 (LKB1), which activates AMPK and AMPK-related kinases (interested readers can refer to [116]) under low-energy conditions, is essential to preserve HSC quiescence [117-119]. The effects of LKB1 on mHSC quiescence might involve the protection of their mitochondria. Indeed, LKB1-deficient mHSCs have reduced mtDNA content, mitochondrial membrane potential, PGC- $1 \alpha$ and $-\beta$ levels, and ATP levels, despite increased mitochondrial mass. These observations suggest that mitochondrial dysfunction occurs in LKB1-deficient mHSCs $[117,118]$. Further studies are required to determine whether this mitochondrial dysfunction is a direct conse- quence of LKB1 inactivation. Examining whether mitochondrial dysfunction plays a direct or indirect role in the disruption of HSC quiescence in LKB1-deficient HSCs is also important.

\section{Metabolites and cofactors: small molecules with a broad influence}

Due to their reliance on different types of metabolisms, PSCs and differentiated cells display distinct metabolomes. A number of metabolites directly modulate gene expression by regulating the activity/stability of transcription factors and by inducing epigenetic modifications [120]. Global chromatin remodeling, characterized by a transition from relatively open chromatin to more compact chromatin, occurs during cell differentiation. Evidence supports a direct role for epigenetic modifications in driving cell fate decisions rather than stabilizing gene expression [121]. In the following sections, we propose several ways in which the differences in metabolomes could affect gene expression and control cell differentiation.

The redox state and Sirtuin proteins. The $\mathrm{NAD}^{+} / \mathrm{NADH}$ ratio, which is dependent on glycolytic and mitochondrial metabolisms, might regulate the cell fate by modulating $\mathrm{NAD}^{+}$-dependent deacetylase enzymes, such as sirtuins. These proteins are involved in the control of gene expression and epigenetics through deacetylation of transcriptional regulators or histone proteins [122]. SIRT1 levels are decreased during hESC and mESC differentiation [123,124]. Although SIRT1 is not required for mHSC function [125], some reports suggest that SIRT1 might be required for both the maintenance and differentiation of mHSCs [126]. In MSCs, SIRT1 activity favors osteogenesis while inhibiting adipogenesis [115]. The accumulated evidence clearly supports the involvement of SIRT1 in the regulation of pluripotency and differentiation processes, although the mechanisms underlying stage and lineage specificities remain obscure. Bidirectional crosstalk between the activity of sirtuins and mitochondrial metabolism might exist as sirtuins can induce mitochondrial biogenesis [127] and regulate mitochondrial energy metabolism through the posttranslational regulation of mitochondrial proteins [128]. Therefore, further studies should assess the putative bidirectional influences of SIRT1 and mitochondrial biogenesis and function in various models of stem cell differentiation.

TCA cycle metabolites: roles inside and outside the mitochondria. Intermediates of the TCA cycle might also be involved in determining cell fate. For example, the $\alpha$ ketoglutarate metabolite can exit mitochondria and promote DNA and histone demethylation through the activation of 10-11 translocation enzymes, lysine demethylases, and Jumonji $\mathrm{C}$ domain-containing demethylases. $\alpha$-Ketoglutarate is also involved in the stability of HIF-1 through the activation of the prolyl hydroxylase domain-containing protein 2 , stimulating the degradation of HIF- $1 \alpha$ [120]. This might be of critical significance in the interplay between mitochondria, metabolism, and pluripotency, given the recently discovered role of HIF- $1 \alpha$ in the coregulation of those processes [70]. Similarly, the acetyl-coenzyme A cofactor, which is mainly produced downstream of glycolysis and fatty acid oxidation, can, instead of feeding the TCA cycle, exit mitochondria and reach the nucleus where it promotes 
histone acetylation, as described in a model of adipocyte differentiation [129].

\section{Noncoding RNAs: a new way to decode the interplay between mitochondria and cell differentiation?}

The role of noncoding RNAs, such as microRNAs (miRNAs) or long noncoding RNAs (lncRNAs) in many biological processes, including stem cell differentiation and metabolism regulation, is increasingly being appreciated. Their broad impact, resulting from their various mechanisms of action, as well as high number of targets, a makes noncoding RNAs appealing candidates for the coregulation of simultaneous processes. On the one hand, miR-1, a myomir induced during myogenic differentiation, was reported to inhibit the expression of a transcriptional repressor of muscle gene expression [130], but on the other hand, it was reported to enter the mitochondria and stimulate the translation of specific mitochondrial genome-encoded transcripts [131]. These data suggest that miR-1 might coordinate the myogenic program by coregulating mitochondrial biogenesis and the myogenic-associated transcriptional program by distinct mechanisms [131]. In a recent study, a single-cell transcriptome analysis of several cell types undergoing reprogramming into iPSCs identified two lncRNAs involved in the regulation of multiple mitochondrial and metabolic targets [132]. A number of noncoding RNAs have reported roles in regulating cell fate or cell metabolism and mitochondrial biogenesis. Future studies should assess the relevance of those candidates as putative global coregulators of cellular fate and mitochondrial biogenesis and function.

\section{Conclusion and Perspectives}

The deep remodeling of mitochondria observed during stem cell differentiation and reprogramming has unveiled a critical role for glycolytic and oxidative metabolisms in the regulation of self-renewal, pluripotency, plasticity, and differentiation of stem cells. PSCs rely heavily on glycolysis, which provides rapid energy production and the substrates necessary for proliferation, is adapted to a hypoxic niche, and enables limited production of ROS. On the other hand, differentiated cells display a more developed and functional mitochondrial network and rely heavily on OXPHOS. Accordingly, mitochondrial function directly regulates stem cell differentiation through various mechanisms involving ROS production, metabolomic modifications, and modulation of the redox and energy status. Many of the pathways involved in the interplay between glycolytic and oxidative metabolisms on the one hand, and pluripotency on the other hand, act bidirectionally and interact with each other. This leads to a complex and still unresolved question: Is the mitochondrial biogenesis initiated first, followed by a loss of pluripotency and a commitment to differentiation, which is further accompanied by increased mitochondrial function? Or does the loss of pluripotency initiate mitochondrial biogenesis and a metabolic shift? Single cell tracking and combined OMICS technologies might help to resolve this question, as well as to further elucidate the underlying pathways. It would be intriguing to study how mitochondria are distributed and how they evolve in self-renewing or com- mitted cells upon asymmetric division. Efforts should be made to study mitochondrial and metabolic changes upon in vivo differentiation. The recent development of genetically encoded fluorescent probes, such as roGFP ${ }^{\text {mito }}$ [133], which can be used to assess the mitochondrial oxidation state, or mitoTimer [134], which can be used to follow mitochondrial protein turnover/segregation, will likely help to address these questions. Given the considerable therapeutic potential of stem cell therapy, improving our knowledge about the mechanisms regulating their self-renewal and differentiation, which may involve mitochondria, is fundamental.

\section{Acknowledgment}

The authors thank the Association Belge contre les Maladies Neuro-Musculaires (ABMM, Belgium) for their support.

\section{Author Disclosure Statement}

The authors declare no conflicts of interest.

\section{References}

1. Zhang $\mathrm{H}$ and ZZ Wang. (2008). Mechanisms that mediate stem cell self-renewal and differentiation. J Cell Biochem 103:709-718.

2. Takahashi K and S Yamanaka. (2006). Induction of pluripotent stem cells from mouse embryonic and adult fibroblast cultures by defined factors. Cell 126:663-676.

3. Teslaa T and MA Teitell. (2015). Pluripotent stem cell energy metabolism: an update. EMBO J 34:138-153.

4. Hock MB and A Kralli. (2009). Transcriptional control of mitochondrial biogenesis and function. Annu Rev Physiol 71:177-203.

5. St John JC, J Ramalho-Santos, HL Gray, P Petrosko, VY Rawe, CS Navara, CR Simerly and GP Schatten. (2005). The expression of mitochondrial DNA transcription factors during early cardiomyocyte in vitro differentiation from human embryonic stem cells. Cloning Stem Cells 7:141-153.

6. Mandal S, AG Lindgren, AS Srivastava, AT Clark and U Banerjee. (2011). Mitochondrial function controls proliferation and early differentiation potential of embryonic stem cells. Stem Cells 29:486-495.

7. Lonergan T, C Brenner and B Bavister. (2006). Differentiation-related changes in mitochondrial properties as indicators of stem cell competence. J Cell Physiol 208: 149-153.

8. Prigione A, B Fauler, R Lurz, H Lehrach and J Adjaye. (2010). The senescence-related mitochondrial/oxidative stress pathway is repressed in human induced pluripotent stem cells. Stem Cells 28:721-733.

9. Varum S, AS Rodrigues, MB Moura, O Momcilovic, CAt Easley, J Ramalho-Santos, B Van Houten and G Schatten. (2011). Energy metabolism in human pluripotent stem cells and their differentiated counterparts. PLoS One 6:e20914.

10. Prowse AB, F Chong, DA Elliott, AG Elefanty, EG Stanley, PP Gray, TP Munro and GW Osborne. (2012). Analysis of mitochondrial function and localisation during human embryonic stem cell differentiation in vitro. PLoS One 7:e52214.

11. Sathananthan H, M Pera and A Trounson. (2002). The fine structure of human embryonic stem cells. Reprod Biomed Online 4:56-61. 
12. Baharvand H and KI Matthaei. (2003). The ultrastructure of mouse embryonic stem cells. Reprod Biomed Online 7:330-335.

13. Oh SK, HS Kim, HJ Ahn, HW Seol, YY Kim, YB Park, CJ Yoon, DW Kim, SH Kim and SY Moon. (2005). Derivation and characterization of new human embryonic stem cell lines: SNUhES1, SNUhES2, and SNUhES3. Stem Cells 23:211-219.

14. Lonergan T, B Bavister and C Brenner. (2007). Mitochondria in stem cells. Mitochondrion 7:289-296.

15. Cho YM, S Kwon, YK Pak, HW Seol, YM Choi, J Park do, KS Park and HK Lee. (2006). Dynamic changes in mitochondrial biogenesis and antioxidant enzymes during the spontaneous differentiation of human embryonic stem cells. Biochem Biophys Res Commun 348:14721478.

16. Folmes CD, TJ Nelson, A Martinez-Fernandez, DK Arrell, JZ Lindor, PP Dzeja, Y Ikeda, C Perez-Terzic and A Terzic. (2011). Somatic oxidative bioenergetics transitions into pluripotency-dependent glycolysis to facilitate nuclear reprogramming. Cell Metab 14:264-271.

17. Facucho-Oliveira JM, J Alderson, EC Spikings, S Egginton and JC St John. (2007). Mitochondrial DNA replication during differentiation of murine embryonic stem cells. J Cell Sci 120:4025-4034.

18. Zhang J, I Khvorostov, JS Hong, Y Oktay, L Vergnes, E Nuebel, PN Wahjudi, K Setoguchi, G Wang, et al. (2011). UCP2 regulates energy metabolism and differentiation potential of human pluripotent stem cells. EMBO J 30: 4860-4873.

19. Panopoulos AD, O Yanes, S Ruiz, YS Kida, D Diep, R Tautenhahn, A Herrerias, EM Batchelder, N Plongthongkum, et al. (2012). The metabolome of induced pluripotent stem cells reveals metabolic changes occurring in somatic cell reprogramming. Cell Res 22:168-177.

20. Pereira SL, M Graos, AS Rodrigues, SI Anjo, RA Carvalho, PJ Oliveira, E Arenas and J Ramalho-Santos. (2013). Inhibition of mitochondrial complex III blocks neuronal differentiation and maintains embryonic stem cell pluripotency. PLoS One 8:e82095.

21. Zhou W, M Choi, D Margineantu, L Margaretha, J Hesson, C Cavanaugh, CA Blau, MS Horwitz, D Hockenbery, C Ware and H Ruohola-Baker. (2012). HIF1alpha induced switch from bivalent to exclusively glycolytic metabolism during ESC-to-EpiSC/hESC transition. EMBO J 31:21032116.

22. Nichols J and A Smith. (2009). Naive and primed pluripotent states. Cell Stem Cell 4:487-492.

23. Takashima Y, G Guo, R Loos, J Nichols, G Ficz, F Krueger, D Oxley, F Santos, J Clarke, et al. (2014). Resetting transcription factor control circuitry toward ground-state pluripotency in human. Cell 158:1254-1269.

24. Choi HW, JH Kim, MK Chung, YJ Hong, HS Jang, BJ Seo, TH Jung, JS Kim, HM Chung, et al. (2015). Mitochondrial and metabolic remodeling during reprogramming and differentiation of the reprogrammed cells. Stem Cells Dev 24:1366-1373.

25. Masotti A, A Celluzzi, S Petrini, E Bertini, G Zanni and C Compagnucci. (2014). Aged iPSCs display an uncommon mitochondrial appearance and fail to undergo in vitro neurogenesis. Aging (Albany NY) 6:1094-1108.

26. Sharma NS, EJ Wallenstein, E Novik, T Maguire, R Schloss and ML Yarmush. (2009). Enrichment of hepatocyte-like cells with upregulated metabolic and dif- ferentiated function derived from embryonic stem cells using S-NitrosoAcetylPenicillamine. Tissue Eng Part C Methods 15:297-306.

27. Kanno S, PK Kim, K Sallam, J Lei, TR Billiar and LL Shears, 2nd. (2004). Nitric oxide facilitates cardiomyogenesis in mouse embryonic stem cells. Proc Natl Acad Sci U S A 101:12277-12281.

28. Villena JA. (2014). New insights into PGC-1 coactivators: redefining their role in the regulation of mitochondrial function and beyond. FEBS J 282:647-672.

29. Huang PI, YC Chou, YL Chang, Y Chien, KH Chen, WS Song, CH Peng, CH Chang, SD Lee, et al. (2011). Enhanced differentiation of three-gene-reprogrammed induced pluripotent stem cells into adipocytes via adenoviralmediated PGC-1alpha overexpression. Int J Mol Sci 12: 7554-7568.

30. Zhu S, W Li, H Zhou, W Wei, R Ambasudhan, T Lin, J Kim, K Zhang and S Ding. (2010). Reprogramming of human primary somatic cells by OCT4 and chemical compounds. Cell Stem Cell 7:651-655.

31. Prasad SM, M Czepiel, C Cetinkaya, K Smigielska, SC Weli, H Lysdahl, A Gabrielsen, K Petersen, N Ehlers, et al. (2009). Continuous hypoxic culturing maintains activation of Notch and allows long-term propagation of human embryonic stem cells without spontaneous differentiation. Cell Prolif 42:63-74.

32. Ezashi T, P Das and RM Roberts. (2005). Low O2 tensions and the prevention of differentiation of hES cells. Proc Natl Acad Sci U S A 102:4783-4788.

33. Zhang Y, G Marsboom, PT Toth and J Rehman. (2013). Mitochondrial respiration regulates adipogenic differentiation of human mesenchymal stem cells. PLoS One 8: e77077.

34. Tormos KV, E Anso, RB Hamanaka, J Eisenbart, J Joseph, B Kalyanaraman and NS Chandel. (2011). Mitochondrial complex III ROS regulate adipocyte differentiation. Cell Metab 14:537-544.

35. Hofmann AD, M Beyer, U Krause-Buchholz, M Wobus, M Bornhauser and G Rodel. (2012). OXPHOS supercomplexes as a hallmark of the mitochondrial phenotype of adipogenic differentiated human MSCs. PLoS One 7: e35160.

36. Huang PI, YC Chen, LH Chen, CC Juan, HH Ku, ST Wang, SH Chiou, GY Chiou, CW Chi, et al. (2011). PGC1alpha mediates differentiation of mesenchymal stem cells to brown adipose cells. J Atheroscler Thromb 18:966-980.

37. Chen CT, YR Shih, TK Kuo, OK Lee and YH Wei. (2008). Coordinated changes of mitochondrial biogenesis and antioxidant enzymes during osteogenic differentiation of human mesenchymal stem cells. Stem Cells 26: 960-968.

38. Sanchez-Arago M, J Garcia-Bermudez, I Martinez-Reyes, F Santacatterina and JM Cuezva. (2013). Degradation of IF1 controls energy metabolism during osteogenic differentiation of stem cells. EMBO Rep 14:638-644.

39. Pietila M, S Palomaki, S Lehtonen, I Ritamo, L Valmu, J Nystedt, S Laitinen, HV Leskela, R Sormunen, et al. (2012). Mitochondrial function and energy metabolism in umbilical cord blood- and bone marrow-derived mesenchymal stem cells. Stem Cells Dev 21:575-588.

40. Wanet A, N Remacle, M Najar, E Sokal, T Arnould, M Najimi and P Renard. (2014). Mitochondrial remodeling in hepatic differentiation and dedifferentiation. Int J Biochem Cell Biol 54:174-185. 
41. Mantel C, S Messina-Graham and HE Broxmeyer. (2010). Upregulation of nascent mitochondrial biogenesis in mouse hematopoietic stem cells parallels upregulation of CD34 and loss of pluripotency: a potential strategy for reducing oxidative risk in stem cells. Cell Cycle 9:20082017.

42. Inoue $\mathrm{S}, \mathrm{S}$ Noda, K Kashima, K Nakada, J Hayashi and $\mathrm{H}$ Miyoshi. (2010). Mitochondrial respiration defects modulate differentiation but not proliferation of hematopoietic stem and progenitor cells. FEBS Lett 584:3402-3409.

43. Ye XQ, Q Li, GH Wang, FF Sun, GJ Huang, XW Bian, SC Yu and GS Qian. (2011). Mitochondrial and energy metabolism-related properties as novel indicators of lung cancer stem cells. Int J Cancer 129:820-831.

44. Shen YA, CY Wang, YT Hsieh, YJ Chen and YH Wei. (2015). Metabolic reprogramming orchestrates cancer stem cell properties in nasopharyngeal carcinoma. Cell Cycle 14:86-98.

45. Pereira SL, AS Rodrigues, MI Sousa, M Correia, T Perestrelo and J Ramalho-Santos. (2014). From gametogenesis and stem cells to cancer: common metabolic themes. Hum Reprod Update 20:924-943.

46. Kondoh H, ME Lleonart, Y Nakashima, M Yokode, M Tanaka, D Bernard, J Gil and D Beach. (2007). A high glycolytic flux supports the proliferative potential of murine embryonic stem cells. Antioxid Redox Signal 9:293299.

47. Holness MJ and MC Sugden. (2003). Regulation of pyruvate dehydrogenase complex activity by reversible phosphorylation. Biochem Soc Trans 31:1143-1151.

48. Takubo K, G Nagamatsu, CI Kobayashi, A NakamuraIshizu, H Kobayashi, E Ikeda, N Goda, Y Rahimi, RS Johnson, et al. (2013). Regulation of glycolysis by Pdk functions as a metabolic checkpoint for cell cycle quiescence in hematopoietic stem cells. Cell Stem Cell 12: $49-61$.

49. Vozza A, G Parisi, F De Leonardis, FM Lasorsa, A Castegna, D Amorese, R Marmo, VM Calcagnile, L Palmieri, et al. (2014). UCP2 transports C4 metabolites out of mitochondria, regulating glucose and glutamine oxidation. Proc Natl Acad Sci U S A 111:960-965.

50. Chung S, DK Arrell, RS Faustino, A Terzic and PP Dzeja. (2010). Glycolytic network restructuring integral to the energetics of embryonic stem cell cardiac differentiation. J Mol Cell Cardiol 48:725-734.

51. Pattappa G, SD Thorpe, NC Jegard, HK Heywood, JD de Bruijn and DA Lee. (2013). Continuous and uninterrupted oxygen tension influences the colony formation and oxidative metabolism of human mesenchymal stem cells. Tissue Eng Part C Methods 19:68-79.

52. Polo JM, S Liu, ME Figueroa, W Kulalert, S Eminli, KY Tan, E Apostolou, M Stadtfeld, Y Li, et al. (2010). Cell type of origin influences the molecular and functional properties of mouse induced pluripotent stem cells. Nat Biotechnol 28:848-855.

53. Otera H and K Mihara. (2011). Molecular mechanisms and physiologic functions of mitochondrial dynamics. J Biochem 149:241-251.

54. Westermann B. (2010). Mitochondrial fusion and fission in cell life and death. Nat Rev Mol Cell Biol 11:872884.

55. Otera H, N Ishihara and K Mihara. (2013). New insights into the function and regulation of mitochondrial fission. Biochim Biophys Acta 1833:1256-1268.
56. Son MY, H Choi, YM Han and YS Cho. (2013). Unveiling the critical role of REX1 in the regulation of human stem cell pluripotency. Stem Cells 31:2374-2387.

57. Vazquez-Martin A, S Cufi, B Corominas-Faja, C OliverasFerraros, L Vellon and JA Menendez. (2012). Mitochondrial fusion by pharmacological manipulation impedes somatic cell reprogramming to pluripotency: new insight into the role of mitophagy in cell stemness. Aging (Albany NY) 4:393-401.

58. Wang L, X Ye, Q Zhao, Z Zhou, J Dan, Y Zhu, Q Chen and L Liu. (2014). Drp1 is dispensable for mitochondria biogenesis in induction to pluripotency but required for differentiation of embryonic stem cells. Stem Cells Dev 23:2422-2434.

59. Todd LR, MN Damin, R Gomathinayagam, SR Horn, AR Means and U Sankar. (2010). Growth factor erv1-like modulates Drp1 to preserve mitochondrial dynamics and function in mouse embryonic stem cells. Mol Biol Cell 21:1225-1236.

60. Huang P, CA Galloway and Y Yoon. (2011). Control of mitochondrial morphology through differential interactions of mitochondrial fusion and fission proteins. PLoS One 6:e20655.

61. Kim B, JS Kim, Y Yoon, MC Santiago, MD Brown and JY Park. (2013). Inhibition of Drp1-dependent mitochondrial division impairs myogenic differentiation. Am J Physiol Regul Integr Comp Physiol 305:R927-R938.

62. Kowno M, K Watanabe-Susaki, H Ishimine, S Komazaki, K Enomoto, Y Seki, YY Wang, Y Ishigaki, N Ninomiya, et al. (2014). Prohibitin 2 regulates the proliferation and lineage-specific differentiation of mouse embryonic stem cells in mitochondria. PLoS One 9:e81552.

63. Shen J, X Liu, WM Yu, J Liu, M Groot Nibbelink, C Guo, T Finkel and CK Qu. (2011). A critical role of mitochondrial phosphatase ptpmt 1 in embryogenesis reveals a mitochondrial metabolic stress-induced differentiation checkpoint in embryonic stem cells. Mol Cell Biol 31: 4902-4916.

64. Yu WM, X Liu, J Shen, O Jovanovic, EE Pohl, SL Gerson, T Finkel, HE Broxmeyer and CK Qu. (2013). Metabolic regulation by the mitochondrial phosphatase PTPMT1 is required for hematopoietic stem cell differentiation. Cell Stem Cell 12:62-74.

65. Kasahara A, S Cipolat, Y Chen, GW Dorn, 2nd and L Scorrano. (2013). Mitochondrial fusion directs cardiomyocyte differentiation via calcineurin and Notch signaling. Science 342:734-737.

66. Mitra K. (2013). Mitochondrial fission-fusion as an emerging key regulator of cell proliferation and differentiation. Bioessays 35:955-964.

67. Goda N and M Kanai. (2012). Hypoxia-inducible factors and their roles in energy metabolism. Int $\mathrm{J}$ Hematol 95: 457-463.

68. Mohyeldin A, T Garzon-Muvdi and A Quinones-Hinojosa. (2010). Oxygen in stem cell biology: a critical component of the stem cell niche. Cell Stem Cell 7:150-161.

69. Brocato J, Y Chervona and M Costa. (2014). Molecular responses to hypoxia-inducible factor 1alpha and beyond. Mol Pharmacol 85:651-657.

70. Mahato B, P Home, G Rajendran, A Paul, B Saha, A Ganguly, S Ray, N Roy, RH Swerdlow and S Paul. (2014). Regulation of mitochondrial function and cellular energy metabolism by protein kinase C-lambda/iota: a novel mode of balancing pluripotency. Stem Cells 32:2880-2892. 
71. Prigione A, N Rohwer, S Hoffmann, B Mlody, K Drews, $\mathrm{R}$ Bukowiecki, K Blumlein, EE Wanker, M Ralser, $\mathrm{T}$ Cramer and J Adjaye. (2014). HIF1alpha modulates cell fate reprogramming through early glycolytic shift and upregulation of PDK1-3 and PKM2. Stem Cells 32:364376.

72. Hsu SH, CT Chen and YH Wei. (2013). Inhibitory effects of hypoxia on metabolic switch and osteogenic differentiation of human mesenchymal stem cells. Stem Cells 31: 2779-2788.

73. Murphy MP. (2009). How mitochondria produce reactive oxygen species. Biochem J 417:1-13.

74. Maryanovich M and A Gross. (2013). A ROS rheostat for cell fate regulation. Trends Cell Biol 23:129-134.

75. Sauer H, G Rahimi, J Hescheler and M Wartenberg. (2000). Role of reactive oxygen species and phosphatidylinositol 3-kinase in cardiomyocyte differentiation of embryonic stem cells. FEBS Lett 476:218-223.

76. Schmelter M, B Ateghang, S Helmig, M Wartenberg and H Sauer. (2006). Embryonic stem cells utilize reactive oxygen species as transducers of mechanical straininduced cardiovascular differentiation. FASEB J 20:11821184.

77. Ateghang B, M Wartenberg, M Gassmann and H Sauer. (2006). Regulation of cardiotrophin-1 expression in mouse embryonic stem cells by HIF-1alpha and intracellular reactive oxygen species. J Cell Sci 119:1043-1052.

78. Buggisch M, B Ateghang, C Ruhe, C Strobel, S Lange, M Wartenberg and H Sauer. (2007). Stimulation of EScell-derived cardiomyogenesis and neonatal cardiac cell proliferation by reactive oxygen species and NADPH oxidase. J Cell Sci 120:885-894.

79. Sharifpanah F, M Wartenberg, M Hannig, HM Piper and H Sauer. (2008). Peroxisome proliferator-activated receptor alpha agonists enhance cardiomyogenesis of mouse ES cells by utilization of a reactive oxygen speciesdependent mechanism. Stem Cells 26:64-71.

80. Cho SW, JS Park, HJ Heo, SW Park, S Song, I Kim, YM Han, JK Yamashita, JB Youm, J Han and GY Koh. (2014). Dual modulation of the mitochondrial permeability transition pore and redox signaling synergistically promotes cardiomyocyte differentiation from pluripotent stem cells. J Am Heart Assoc 3:e000693.

81. Tsatmali M, EC Walcott and KL Crossin. (2005). Newborn neurons acquire high levels of reactive oxygen species and increased mitochondrial proteins upon differentiation from progenitors. Brain Res 1040:137-150.

82. Hou Y, X Ouyang, R Wan, H Cheng, MP Mattson and A Cheng. (2012). Mitochondrial superoxide production negatively regulates neural progenitor proliferation and cerebral cortical development. Stem Cells 30:2535-2547.

83. Hou Y, MP Mattson and A Cheng. (2013). Permeability transition pore-mediated mitochondrial superoxide flashes regulate cortical neural progenitor differentiation. PLoS One 8:e76721.

84. Walton NM, R Shin, K Tajinda, CL Heusner, JH Kogan, S Miyake, Q Chen, K Tamura and M Matsumoto. (2012). Adult neurogenesis transiently generates oxidative stress. PLoS One 7:e35264.

85. Kim JH, SH Kim, SY Song, WS Kim, SU Song, T Yi, MS Jeon, HM Chung, Y Xia and JH Sung. (2014). Hypoxia induces adipocyte differentiation of adipose-derived stem cells by triggering reactive oxygen species generation. Cell Biol Int 38:32-40.
86. Wu J, J Niu, X Li, X Wang, Z Guo and F Zhang. (2014). TGF-beta1 induces senescence of bone marrow mesenchymal stem cells via increase of mitochondrial ROS production. BMC Dev Biol 14:21.

87. Kohli L and E Passegue. (2014). Surviving change: the metabolic journey of hematopoietic stem cells. Trends Cell Biol 24:479-487.

88. Jang YY and SJ Sharkis. (2007). A low level of reactive oxygen species selects for primitive hematopoietic stem cells that may reside in the low-oxygenic niche. Blood 110:3056-3063.

89. Ito $\mathrm{K}$, A Hirao, $\mathrm{F}$ Arai, $\mathrm{K}$ Takubo, $\mathrm{S}$ Matsuoka, $\mathrm{K}$ Miyamoto, M Ohmura, K Naka, K Hosokawa, Y Ikeda and T Suda. (2006). Reactive oxygen species act through p38 MAPK to limit the lifespan of hematopoietic stem cells. Nat Med 12:446-451.

90. Owusu-Ansah E and U Banerjee. (2009). Reactive oxygen species prime Drosophila haematopoietic progenitors for differentiation. Nature 461:537-541.

91. Juntilla MM, VD Patil, M Calamito, RP Joshi, MJ Birnbaum and GA Koretzky. (2010). AKT1 and AKT2 maintain hematopoietic stem cell function by regulating reactive oxygen species. Blood 115:4030-4038.

92. Hamanaka RB, A Glasauer, P Hoover, S Yang, H Blatt, AR Mullen, S Getsios, CJ Gottardi, RJ DeBerardinis, RM Lavker and NS Chandel. (2013). Mitochondrial reactive oxygen species promote epidermal differentiation and hair follicle development. Sci Signal 6:ra8.

93. Campbell CT, JE Kolesar and BA Kaufman. (2012). Mitochondrial transcription factor A regulates mitochondrial transcription initiation, DNA packaging, and genome copy number. Biochim Biophys Acta 1819:921-929.

94. Laplante M and DM Sabatini. (2009). mTOR signaling at a glance. J Cell Sci 122:3589-3594.

95. Laplante M and DM Sabatini. (2012). mTOR signaling in growth control and disease. Cell 149:274-293.

96. Murakami M, T Ichisaka, M Maeda, N Oshiro, K Hara, F Edenhofer, H Kiyama, K Yonezawa and S Yamanaka. (2004). mTOR is essential for growth and proliferation in early mouse embryos and embryonic stem cells. Mol Cell Biol 24:6710-6718.

97. Zhou J, P Su, L Wang, J Chen, M Zimmermann, O Genbacev, O Afonja, MC Horne, T Tanaka, et al. (2009). mTOR supports long-term self-renewal and suppresses mesoderm and endoderm activities of human embryonic stem cells. Proc Natl Acad Sci U S A 106:7840-7845.

98. Schieke SM, M Ma, L Cao, JP McCoy, Jr., C Liu, NF Hensel, AJ Barrett, M Boehm and T Finkel. (2008). Mitochondrial metabolism modulates differentiation and teratoma formation capacity in mouse embryonic stem cells. J Biol Chem 283:28506-28512.

99. Agrawal P, J Reynolds, S Chew, DA Lamba and RE Hughes. (2014). DEPTOR is a stemness factor that regulates pluripotency of embryonic stem cells. J Biol Chem 289:31818-31826.

100. Wyatt LA, MT Filbin and HS Keirstead. (2014). PTEN inhibition enhances neurite outgrowth in human embryonic stem cell-derived neuronal progenitor cells. J Comp Neurol 522:2741-2755.

101. Easley CAt, A Ben-Yehudah, CJ Redinger, SL Oliver, ST Varum, VM Eisinger, DL Carlisle, PJ Donovan and GP Schatten. (2010). mTOR-mediated activation of p70 S6K induces differentiation of pluripotent human embryonic stem cells. Cell Reprogram 12:263-273. 
102. Chen T, L Shen, J Yu, H Wan, A Guo, J Chen, Y Long, J Zhao and G Pei. (2011). Rapamycin and other longevitypromoting compounds enhance the generation of mouse induced pluripotent stem cells. Aging Cell 10:908-911.

103. He J, L Kang, T Wu, J Zhang, H Wang, H Gao, Y Zhang, B Huang, W Liu, et al. (2012). An elaborate regulation of Mammalian target of rapamycin activity is required for somatic cell reprogramming induced by defined transcription factors. Stem Cells Dev 21:2630-2641.

104. Chen C, Y Liu, R Liu, T Ikenoue, KL Guan and P Zheng. (2008). TSC-mTOR maintains quiescence and function of hematopoietic stem cells by repressing mitochondrial biogenesis and reactive oxygen species. J Exp Med 205: 2397-2408.

105. Kalaitzidis D, SM Sykes, Z Wang, N Punt, Y Tang, C Ragu, AU Sinha, SW Lane, AL Souza, et al. (2012). mTOR complex 1 plays critical roles in hematopoiesis and Ptenloss-evoked leukemogenesis. Cell Stem Cell 11:429-439.

106. Shirwany NA and MH Zou. (2014). AMPK: a cellular metabolic and redox sensor. A minireview. Front Biosci (Landmark Ed) 19:447-474.

107. Prigione A, B Lichtner, H Kuhl, EA Struys, M Wamelink, $\mathrm{H}$ Lehrach, M Ralser, B Timmermann and J Adjaye. (2011). Human induced pluripotent stem cells harbor homoplasmic and heteroplasmic mitochondrial DNA mutations while maintaining human embryonic stem celllike metabolic reprogramming. Stem Cells 29:1338-1348.

108. Vazquez-Martin A, L Vellon, PM Quiros, S Cufi, E Ruiz de Galarreta, C Oliveras-Ferraros, AG Martin, B MartinCastillo, C Lopez-Otin and JA Menendez. (2012). Activation of AMP-activated protein kinase (AMPK) provides a metabolic barrier to reprogramming somatic cells into stem cells. Cell Cycle 11:974-989.

109. Vazquez-Martin A, B Corominas-Faja, S Cufi, L Vellon, C Oliveras-Ferraros, OJ Menendez, J Joven, R Lupu and JA Menendez. (2013). The mitochondrial H(+)-ATP synthase and the lipogenic switch: new core components of metabolic reprogramming in induced pluripotent stem (iPS) cells. Cell Cycle 12:207-218.

110. Shi X, Y Wu, Z Ai, X Liu, L Yang, J Du, J Shao, Z Guo and Y Zhang. (2013). AICAR sustains J1 mouse embryonic stem cell self-renewal and pluripotency by regulating transcription factor and epigenetic modulator expression. Cell Physiol Biochem 32:459-475.

111. Williamson DL, DC Butler and SE Alway. (2009). AMPK inhibits myoblast differentiation through a PGC-1alphadependent mechanism. Am J Physiol Endocrinol Metab 297:E304-E314.

112. Li X, Y Han, W Pang, C Li, X Xie, JY Shyy and Y Zhu. (2008). AMP-activated protein kinase promotes the differentiation of endothelial progenitor cells. Arterioscler Thromb Vasc Biol 28:1789-1795.

113. Dzeja PP, S Chung, RS Faustino, A Behfar and A Terzic. (2011). Developmental enhancement of adenylate kinaseAMPK metabolic signaling axis supports stem cell cardiac differentiation. PLoS One 6:e19300.

114. Padmasekar M, F Sharifpanah, A Finkensieper, M Wartenberg and H Sauer. (2011). Stimulation of cardiomyogenesis of embryonic stem cells by nitric oxide downstream of AMP-activated protein kinase and mTOR signaling pathways. Stem Cells Dev 20:2163-2175.

115. Chen H, X Liu, J Cao, L Zhang, X Hu and J Wang. (2014). Role of SIRT1 and AMPK in mesenchymal stem cells differentiation. Ageing Res Rev 13:55-64.
116. Bright NJ, C Thornton and D Carling. (2009). The regulation and function of mammalian AMPK-related kinases. Acta Physiol (Oxf) 196:15-26.

117. Gan B, J Hu, S Jiang, Y Liu, E Sahin, L Zhuang, E Fletcher-Sananikone, S Colla, YA Wang, L Chin and RA Depinho. (2010). Lkb1 regulates quiescence and metabolic homeostasis of haematopoietic stem cells. Nature 468:701-704.

118. Nakada D, TL Saunders and SJ Morrison. (2010). Lkb1 regulates cell cycle and energy metabolism in haematopoietic stem cells. Nature 468:653-658.

119. Gurumurthy S, SZ Xie, B Alagesan, J Kim, RZ Yusuf, B Saez, A Tzatsos, F Ozsolak, P Milos, et al. (2010). The Lkb1 metabolic sensor maintains haematopoietic stem cell survival. Nature 468:659-663.

120. Meier JL. (2013). Metabolic mechanisms of epigenetic regulation. ACS Chem Biol 8:2607-2621.

121. Chen T and SY Dent. (2014). Chromatin modifiers and remodellers: regulators of cellular differentiation. Nat Rev Genet 15:93-106.

122. Houtkooper RH, E Pirinen and J Auwerx. (2012). Sirtuins as regulators of metabolism and healthspan. Nat Rev Mol Cell Biol 13:225-238.

123. Saunders LR, AD Sharma, J Tawney, M Nakagawa, K Okita, S Yamanaka, H Willenbring and E Verdin. (2010). miRNAs regulate SIRT1 expression during mouse embryonic stem cell differentiation and in adult mouse tissues. Aging (Albany NY) 2:415-431.

124. Calvanese V, E Lara, B Suarez-Alvarez, R Abu Dawud, M Vazquez-Chantada, ML Martinez-Chantar, N Embade, P Lopez-Nieva, A Horrillo, et al. (2010). Sirtuin 1 regulation of developmental genes during differentiation of stem cells. Proc Natl Acad Sci U S A 107:1373613741.

125. Leko V, B Varnum-Finney, H Li, Y Gu, D Flowers, C Nourigat, ID Bernstein and A Bedalov. (2012). SIRT1 is dispensable for function of hematopoietic stem cells in adult mice. Blood 119:1856-1860.

126. Matsui K, S Ezoe, K Oritani, M Shibata, M Tokunaga, N Fujita, A Tanimura, T Sudo, H Tanaka, et al. (2012). NAD-dependent histone deacetylase, SIRT1, plays essential roles in the maintenance of hematopoietic stem cells. Biochem Biophys Res Commun 418:811-817.

127. Brenmoehl J and A Hoeflich. (2013). Dual control of mitochondrial biogenesis by sirtuin 1 and sirtuin 3. Mitochondrion 13:755-761.

128. Osborne B, GJ Cooney and N Turner. (2014). Are sirtuin deacylase enzymes important modulators of mitochondrial energy metabolism? Biochim Biophys Acta 1840:12951302.

129. Wellen KE, G Hatzivassiliou, UM Sachdeva, TV Bui, JR Cross and CB Thompson. (2009). ATP-citrate lyase links cellular metabolism to histone acetylation. Science 324: 1076-1080.

130. Chen JF, EM Mandel, JM Thomson, Q Wu, TE Callis, SM Hammond, FL Conlon and DZ Wang. (2006). The role of microRNA-1 and microRNA-133 in skeletal muscle proliferation and differentiation. Nat Genet 38:228-233.

131. Zhang X, X Zuo, B Yang, Z Li, Y Xue, Y Zhou, J Huang, X Zhao, J Zhou, et al. (2014). MicroRNA directly enhances mitochondrial translation during muscle differentiation. Cell 158:607-619.

132. Kim DH, GK Marinov, S Pepke, ZS Singer, P He, B Williams, GP Schroth, MB Elowitz and BJ Wold. (2015). 
Single-cell transcriptome analysis reveals dynamic changes in lncRNA expression during reprogramming. Cell Stem Cell 16:88-101.

133. Soberanes S, D Urich, CM Baker, Z Burgess, SE Chiarella, EL Bell, AJ Ghio, A De Vizcaya-Ruiz, J Liu, et al. (2009). Mitochondrial complex III-generated oxidants activate ASK1 and JNK to induce alveolar epithelial cell death following exposure to particulate matter air pollution. J Biol Chem 284:2176-2186.

134. Ferree AW, K Trudeau, E Zik, IY Benador, G Twig, RA Gottlieb and OS Shirihai. (2013). MitoTimer probe reveals the impact of autophagy, fusion, and motility on subcellular distribution of young and old mitochondrial protein and on relative mitochondrial protein age. Autophagy 9:1887-1896.
Address correspondence to:

Prof. Patricia Renard

Laboratory of Biochemistry and Cell Biology (URBC) NAmur Research Institute for LIfe Sciences (NARILIS)

University of Namur (UNamur)

61 rue de Bruxelles Namur 5000

Belgium

E-mail: patsy.renard@unamur.be

Received for publication March 29, 2015

Accepted after revision May 26, 2015

Prepublished on Liebert Instant Online May 28, 2015 